\title{
Pyogenic Flexor Tenosynovitis Caused by Shewanella putrefaciens
}

\author{
Anooj Patel ${ }^{1}$, Mona Ascha ${ }^{2}$, Ayesha Punjabi ${ }^{3}$, Marco Swanson ${ }^{3}$, Tobias C. Long 2,3 \\ 1. Plastic Surgery, Case Western Reserve University School of Medicine, Cleveland, USA 2. Plastic Surgery, University \\ Hospitals Cleveland Medical Center, Cleveland, USA 3. Plastic Surgery, University Hospitals, Cleveland, USA
}

Corresponding author: Anooj Patel, axp607@case.edu

\begin{abstract}
Flexor tenosynovitis is a surgical emergency due to the risk of tendon necrosis which can lead to subsequent amputation. We report a case of flexor tenosynovitis with Shewanella putrefaciens as the implicated organism, though the patient's mechanism of penetrating trauma did not involve a marine exposure. Shewanella are Gram negative bacilli associated with marine environments and have rarely been implicated in human disease. This patient presented with all four of Kanavel's signs and required open surgical irrigation and debridement; he was found to have purulence but no flexor tendon necrosis. This case emphasizes the importance of considering marine organisms as putative for flexor tenosynovitis, even if marine exposure does not occur at the time of the penetrating trauma. It also emphasizes the need to obtain a thorough patient history, especially in cases of infection, to assess for all possible environmental exposures.
\end{abstract}

Categories: Plastic Surgery, Infectious Disease, Orthopedics

Keywords: dip: distal interphalangeal, flexor tenosynovitis, shewanella putrefaciens

\section{Introduction}

Flexor tenosynovitis is a closed-space infection of the flexor tendon sheath of the digit. The natural history of this condition is a direct result of the anatomy of the tendon sheath, a double-walled structure consisting of a visceral layer densely adherent to the tendon and a parietal layer adjacent to the pulley system [1]. Between these layers is synovial fluid, which is the first nutritional supply to the flexor tendon; the second is the vincular system. A closed system occurs because these visceral and parietal layers are connected at the proximal and distal extents of the tendon sheath: at the level of the metacarpal neck and just proximal to the distal interphalangeal (DIP) joint, respectively.

Upon inoculating the flexor tendon sheath, bacteria utilize the synovial fluid as a nutritional source and proliferate readily, as the poor vascular supply within the closed space limits the host defense. The closed nature of the tendon sheath also results in profound increases in volume and pressure, which in turn

Received 04/16/2020

Review began 04/16/2020 Review ended 04/27/2020 Published 05/14/2020

\section{() Copyright 2020}

Patel et al. This is an open access article distributed under the terms of the Creative Commons Attribution License CC-BY 4.0., which permits unrestricted use, distribution, and reproduction in any medium, provided the original author and source are credited. obstructs nutritional supply to the tendon via the vincular system [2]. The tendon is thus deprived of both of its nutritional sources, and imminently susceptible to necrosis, rupture, and contracture. For these reasons, flexor tenosynovitis represents a surgical emergency [3]. Typical microbiology in flexor tenosynovitis includes Staphylococcus aureus, beta-hemolytic Streptococcus, or Pasturella multicoda in cases involving animal bites; Mycobacterium and Vibrio species are most commonly associated with water exposure [3-4].

We present the unusual case of a Shewanella putrefaciens flexor tenosynovitis resulting from penetrating trauma via a drill bit in a patient who owns a puffer fish and maintains a fish tank. To our knowledge, Shewanella species (Shewanella algae) has only been implicated in flexor tenosynovitis once prior in the literature, and this was associated with a fish hook injury [4].

\section{Case Presentation}

A 38-year-old right-handed male presented to the ER for left index finger pain and edema. Four days prior, the patient sustained a penetrating injury to the left index finger volar DIP crease via a new, clean drill bit which he was using at home. The patient did not seek medical care at that time, washed the wound with peroxide, applied bacitracin, and bandaged himself. Over the next few days, he experienced worsening pain and swelling. The day before presentation, the patient sustained further trauma to the finger when it caught in a door hinge. Hand surgery was called due to concern for flexor tenosynovitis. The patient endorsed no symptoms other than finger pain. Significant history for the presenting complaint included a prior left thumb dislocation and bilateral carpal tunnel releases.

Past medical history included a history of military service causing traumatic brain injury, depression, anxiety, migraines, fibromyalgia, and arthritis. Past surgical history included a left neck melanoma resection. The patient lives on a farm and works as a semi-retired mechanic; he has sustained many lacerations to his hands bilaterally due to his work, and works in the dirt frequently. The patient takes 


\section{Cureus}

medications for depression and anxiety and has no known allergies. The patient is a former smoker and occasional drinker, and denies illicit drug use. The patient uses well water at home, denies any recent water exposure including rivers, ponds, or oceans, however, did admit to having a fish tank and a puffer fish at home.

On physical exam, the patient was afebrile and in no distress. There was a small puncture wound on the volar DIP crease of the left index finger (Figure 1). No pus or drainage could be expressed from the wound. All four Kanavel signs were present: 1) pain on passive extension, 2) the digit was held in a flexed posture, 3) fusiform swelling of the digit, and 4) tenderness to palpation from the A1 pulley to the DIP crease. Range of motion was limited secondary to pain. Both of the patient's hands were dirty with many scattered small scars from prior injuries from work. The remainder of the hand exam was unremarkable. Basic metabolic panel and complete blood count were within normal limits. Left hand X-ray demonstrated soft tissue swelling of the left index finger without evidence of acute fracture or dislocation.

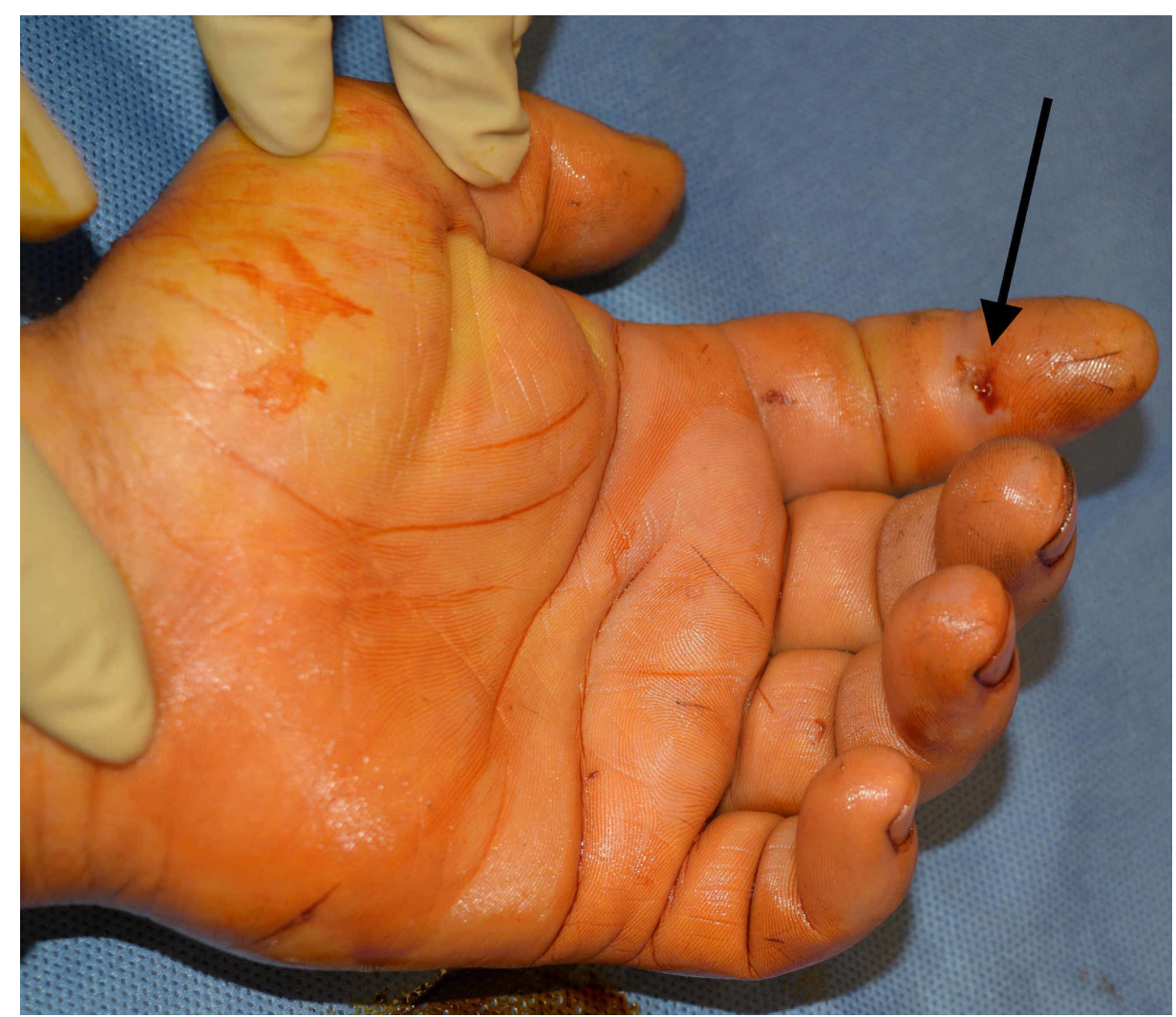

FIGURE 1: Preoperative photograph of left index finger demonstrating site of penetrating injury.

Black arrow points to the exact location of the penetrating injury.

The patient was given a tetanus booster, started on empiric vancomycin and piperacillin/tazobactam, and taken to the operating room emergently. The flexor tendon sheath was approached via a Bruner incision at the volar distal phalanx. Gross purulence was expressed from the distal end of the flexor tendon sheath, confirming the diagnosis of flexor tenosynovitis. A counter-incision in the palm enabled evaluation of the A1 pulley; gross purulence was also expressed at the proximal end of the A1 pulley, and it was subsequently released. Cultures were sent from both proximal and distal sites. The flexor tendon sheath was copiously irrigated and a pediatric feeding tube was placed in the tendon sheath and continuously irrigated with $60 \mathrm{cc}$ of $2 \%$ lidocaine in a liter of normal saline for $48 \mathrm{~h}$. Please refer to Figure $2 A, B$ for the relevant anatomy in pyogenic flexor tenosynovitis. The skin flaps were loosely tacked down with 3-0 nylon suture (Figure 3). 


\section{Cureus}

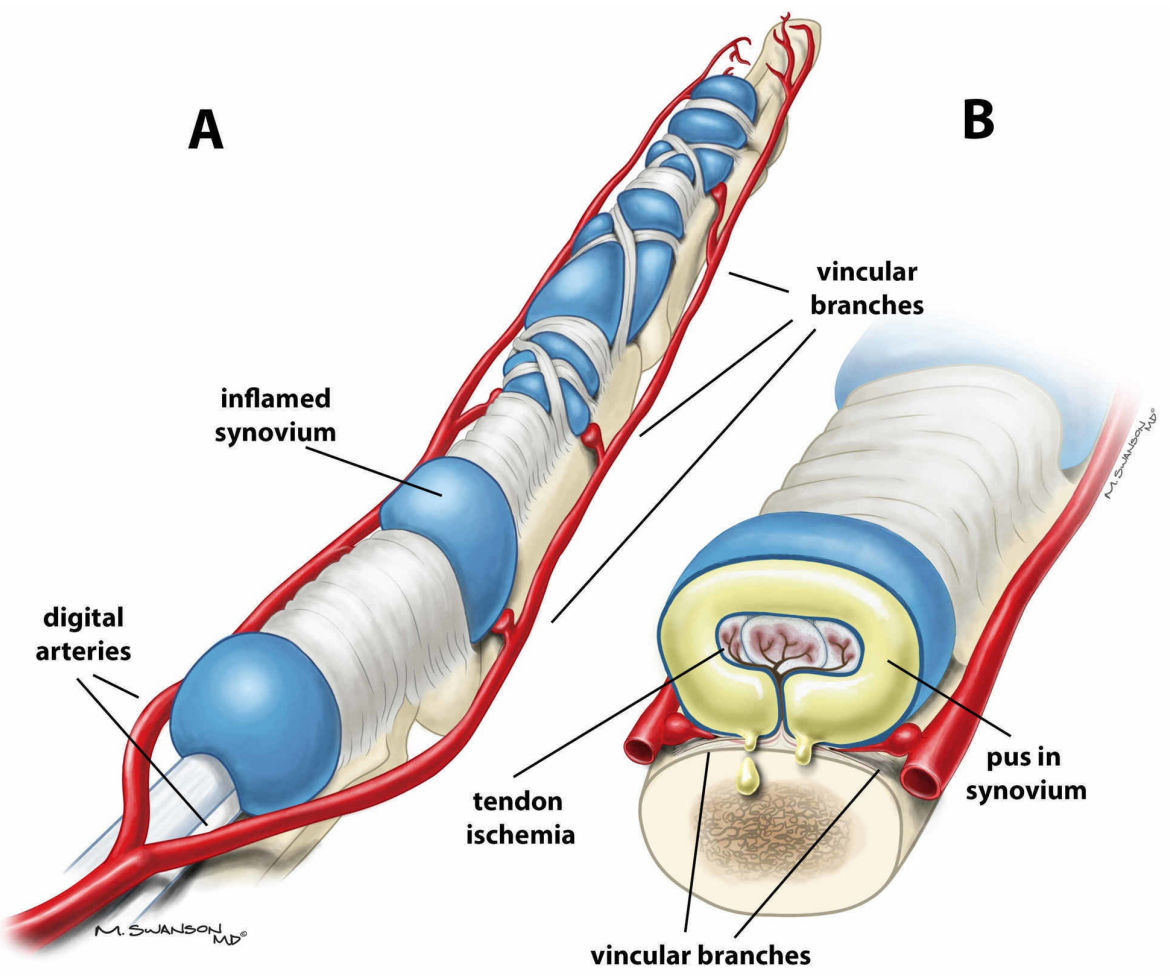

FIGURE 2: A) Anatomy of flexor tendon sheath with swollen sheath due to pyogenic flexor tenosynovitis. B) Cross section of flexor tendon sheath demonstrating pus in the sheath obstructing blood flow from the vincular branches.

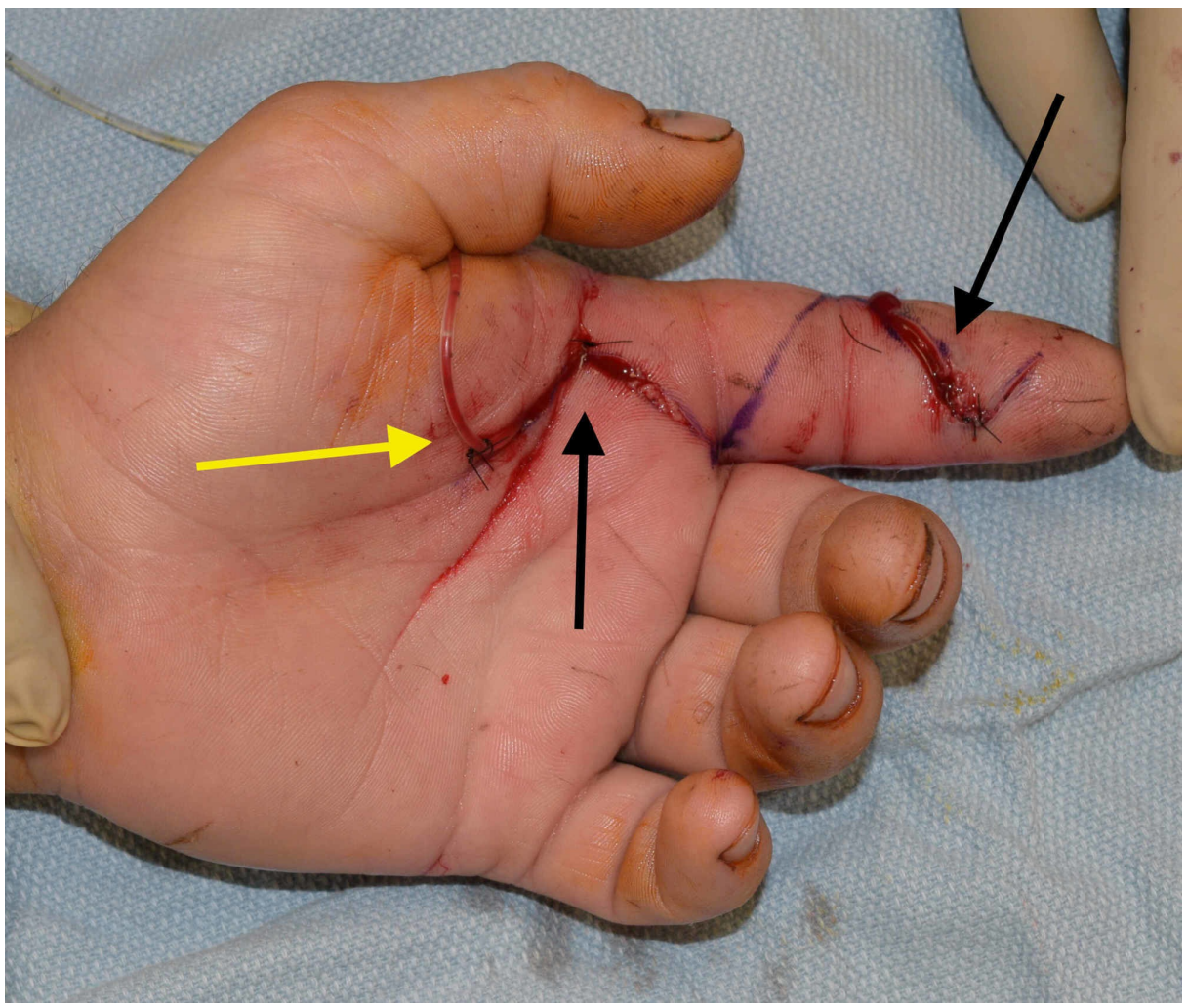

FIGURE 3: Postoperative photograph of left index finger demonstrating 


\section{Cureus}

distal and proximal Bruner's incisions with irrigation catheter placed in flexor tendon sheath.

Black arrows point to the distal and proximal Bruner's incisions. Yellow arrow points to catheter insertion into flexor tendon sheath.

Postoperatively, the patient's symptoms improved. On postoperative day one, distal tendon sheath cultures grew methicillin-sensitive $S$. aureus, Klebsiella oxytoca, and S. putrefaciens, the latter of which was thought to be related to fish tank and puffer fish exposure (Table 1). Proximal tendon sheath cultures grew S. aureus (Table 2). On postoperative day two, the irrigation catheter and dressing were removed, the infectious disease team was consulted, and the occupational therapy was initiated. On postoperative day three, the patient was discharged home with trimethoprim/sulfamethoxazole double strength 800-160 mg oral tab BID for 14 days to treat $S$. aureus and $K$. oxytoca, and ciprofloxacin $500 \mathrm{mg}$ BID for 14 days to treat the $S$. putrefaciens. Further sensitivity testing for $S$. putrefaciens revealed sensitivity to trimethoprim/sulfamethoxazole, and the patient was switched to monotherapy to treat all three organisms.

\begin{tabular}{|c|c|c|c|}
\hline Antibiotic & Shewanella putrefaciens & Klebsiella oxytoca & Staphylococcus aureus \\
\hline Gentamycin & Sensitive & Sensitive & Sensitive \\
\hline Cefepime & Sensitive & & \\
\hline Ceftazidime & Sensitive & & \\
\hline Ciprofloxacin & Sensitive & Sensitive & Sensitive \\
\hline Tobramycin & Sensitive & & \\
\hline Piperacillin/Tazobactam & Sensitive & Sensitive & \\
\hline Trimethoprim/Sulfamethoxazole & Sensitive & Sensitive & Sensitive \\
\hline Ampicillin & & Resistant & Resistant \\
\hline Amoxicillin/Clavulanate & & Sensitive & \\
\hline Cefazolin & & Sensitive & \\
\hline Levofloxacin & & Sensitive & Sensitive \\
\hline Clindamycin & & & Sensitive \\
\hline Erythromycin & & & Sensitive \\
\hline Oxacillin & & & Sensitive \\
\hline Penicillin & & & Resistant \\
\hline Tetracycline & & & Sensitive \\
\hline Vancomycin & & & Sensitive \\
\hline
\end{tabular}

TABLE 1: Cultures and sensitivities from distal tendon sheath. 


\section{Cureus}

\begin{tabular}{|l|l|}
\hline Antibiotic & Staphylococcus aureus \\
\hline Ampicillin & Resistant \\
\hline Clindamycin & Sensitive \\
Ciprofloxacin & Sensitive \\
Erythromycin & Sensitive \\
Gentamicin & Sensitive \\
Levofloxacin & Sensitive \\
\hline Oxacillin & Sensitive \\
\hline Penicillin & Resistant \\
Trimethoprim/Sulfamethoxazole & Sensitive \\
\hline Tetracycline & Sensitive \\
\hline Vancomycin & Sensitive \\
\hline
\end{tabular}

TABLE 2: Cultures and sensitivities from proximal tendon sheath.

On the day of discharge, the patient was afebrile, with significant improvement of finger swelling and pain. Range of motion was improved but still limited due to stiffness. The patient was advised to follow up in clinic in three weeks. At the follow up appointment, the patient's incisions were healed, the antibiotic course was completed, and finger flexion had almost fully returned. He will continue occupational therapy for range of motion.

\section{Discussion}

Shewanella is a nonfermenting Gram negative rod naturally present in marine water and soil; it was initially isolated at the beginning of the twentieth century from putrefied butter and has thereafter also been isolated from fish, sewage, and carcasses. Out of the thirty species of Shewanella, only two (S. putrefaciens and $S$. algae) have been implicated in human diseases, including bacteremia, cellulitis, necrotizing fasciitis, otitis media, open lower extremity fractures, spondylodiscitis, and pneumonia [5-11]. Though flexor tenosynovitis secondary to $S$. algae has been described, and $S$. putrefaciens has been implicated in soft tissue infections of the hand [4,12-13], to our knowledge this is the first reported case of $S$. putrefaciens flexor tenosynovitis caused by a penetrating injury.

Of note, S. algae flexor tenosynovitis presents with a much more rapid course when compared to Mycobacterium marinum, the more common pathogen in flexor tenosynovitis associated with marine exposures [4]. The authors of this case report delineate the severity of flexor tenosynovitis at presentation via Michon's progression [14]:

Stage I: serous exudative fluid with viable tendon

Stage II: purulent fluid with viable tendon

Stage III: septic necrosis of the tendon and pulleys

The reported case of S. algae flexor tenosynovitis presented at stage III, is shown by comparison in Table 3, while M. marinum has been noted to present at stage I [4, 15]. Our patient presented at Michon stage II, suggesting that S. putrefaciens may be intermediate in virulence between S. algae and M. marinum. This is consistent with the suggestion that $S$. algae is the most virulent species of the Shewanella genus [16]. M. marinum, meanwhile, follows a more indolent clinical course, with an incubation period of several weeks. 


\section{Cureus}

\begin{tabular}{|c|c|c|}
\hline & Shewanella putrefaciens case & Shewanella algae case \\
\hline \multicolumn{3}{|l|}{ Kanavel signs } \\
\hline Fusiform swelling & Yes & Yes \\
\hline Finger in flexion & Yes & Yes \\
\hline Pain on passive extension & Yes & Yes \\
\hline Flexor sheath tenderness & Yes & Yes \\
\hline Fever & No & Yes \\
\hline Elevated white blood cell count & No & Yes \\
\hline \multicolumn{3}{|l|}{ Risk factors assoclated with poor outcome } \\
\hline Age $>43$ years & No & No \\
\hline $\begin{array}{l}\text { Presence of diabetes mellitus, peripheral vascular disease, or renal } \\
\text { failure }\end{array}$ & No & No \\
\hline Subcutaneous purulence & Yes & Yes \\
\hline Digital ischemia & No & Yes \\
\hline Polymicrobial infection & Yes & Yes \\
\hline \multicolumn{3}{|l|}{ Michon classification } \\
\hline Stage I & No & No \\
\hline Stage II & Yes & No \\
\hline Stage III & No & Yes \\
\hline
\end{tabular}

TABLE 3: Classification comparison between our case: Shewanella putrefaciens, and an existing case: Shewanella algae.

Table includes data adapted from a case report on Shewanella algae [4].

The classification system proposed by Pang et al delineates risk for poor outcomes in flexor tenosynovitis, particularly digit amputation [17]. The following factors were found to be significant:

1. age greater than 43 years

2. presence of diabetes mellitus

3. peripheral vascular disease or renal failure

4. subcutaneous purulence

5. digital ischemia

6. polymicrobial infection

The fourth and sixth risk factors were applicable to our patient.

Shewanella has demonstrated resistance to penicillin, but susceptibility to ampicillin-sulbactam, piperacillin-tazobactam (which our patient received), cephalosporins, aminoglycosides, and fluoroquinolones [7]. Fortunately for our patient, all three organisms implicated in his infection were sensitive to trimethoprim-sulfamethoxazole.

Unique aspects of our case include $S$. putrefaciens as the putative organism in flexor tenosynovitis, and the disconnect between the mechanism of penetrating injury to the flexor surface of the finger and the marine exposure. Our patient's penetrating injury was with a clean drill bit, and likely exposure to Shewanella was 
from caring for his puffer fish. It is unclear whether Shewanella had previously colonized the skin flora exposed to the penetrating injury or if the exposure was afterwards. This emphasizes that marine organisms including Shewanella should be considered for any marine exposure in flexor tenosynovitis, even if separates from the penetrating trauma. In addition, a thorough history should be obtained at the time of injury.

\section{Conclusions}

Flexor tenosynovitis is a closed-space infection of the flexor tendon that requires urgent diagnosis and treatment with both surgery and antibiotics. The high risk of tendon necrosis can lead to amputation of the digit if not treated correctly. Organisms that have been commonly known to inoculate the sheath after a penetrating injury in various settings include $S$. aureus, beta-hemolytic Streptococcus, P. multicoda, Mycobacterium, and the Vibrio species. Rarer species may also be involved, and thus a detailed patient history is important to start appropriate treatment. This case highlights the importance of broadening the possible infectious microbiology to include Shewanella, when patients have a history of exposure to marine organisms, even if the exposure is not at the time of the penetrating injury. Shewanella algae may be more virulent in comparison to $S$. putrefaciens.

\section{Additional Information \\ Disclosures}

Human subjects: Consent was obtained by all participants in this study. Conflicts of interest: In compliance with the ICMJE uniform disclosure form, all authors declare the following: Payment/services info: All authors have declared that no financial support was received from any organization for the submitted work. Financial relationships: All authors have declared that they have no financial relationships at present or within the previous three years with any organizations that might have an interest in the submitted work. Other relationships: All authors have declared that there are no other relationships or activities that could appear to have influenced the submitted work.

\section{References}

1. Doyle JR: Anatomy of the finger flexor tendon sheath and pulley system . J Hand Surg Am. 1988, 13:473-484.

2. Schnall SB, Vu-rose T, Holtom PD, Doyle B, Stevanovic M: Tissue pressures in pyogenic flexor tenosynovitis of the finger. Compartment syndrome and its management. J Bone Joint Surg Br. 1996, 78:793-795.

3. Wolfe S, Pederson W, Kozin SH, Cohen M: Acute infections of the hand, Chapter 2 . Green's Operative Hand Surgery, Seventh Edition. Elsevier, Amsterdam; 2016. 17-61.

4. Fluke EC, Carayannopoulos NL, Lindsey RW: Pyogenic flexor tenosynovitis caused by Shewanella algae . J Hand Surg Am. 2016, 41:203-206.

5. Vignier N, Barreau M, Olive C, Baubion E, Théodose R, Hochedez P, Cabié A: Human infection with Shewanella putrefaciens and S. algae: report of 16 cases in martinique and review of the literature. Am J Trop Med Hyg. 2013, 89:151-156.

6. Chen YS, Liu YC, Yen MY, et al.: Skin and soft-tissue manifestations of Shewanella putrefaciens infection. Clin Infect Dis. 1997, 25:225-229.

7. Jorens PG, Goovaerts K, Leven M: Shewanella putrefaciens isolated in a case of ventilator-associated pneumonia. Respiration. 2004, 71:199-201.

8. Raja M, Gonzales Zamora JA, Roig I: Shewanella cellulitis and bacteremia following marine water exposure. ID Cases. 2018, 12:140-141.

9. Gressier M, Mbayo D, Deramond H, Grados F, Eb F, Canarelli B: First case of human spondylodiscitis due to Shewanella algae. Int J Infect Dis. 2010, 14:261-264.

10. Sharma KK, Kalawat U: Emerging infections: shewanella - a series of five cases . J Lab Phys. 2010, 2:61-65. 10.4103/0974-2727.72150

11. Smith JR, Morgan M, Palmer JH: Shewanella algae infection complicating an open lower limb fracture . J Plast Reconstr Aesthet Surg. 2014, 67:99-100.

12. Ryan JM, Truelove E, Sabatino M, Peters S, Kessler M: Palmar soft tissue infection from Shewanella putrefaciens. J Hand Surg Am. 2018, 43:87-91.

13. Leong J, Mirkazemi M, Kimble F: Shewanella putrefaciens hand infection. Aust NZ J Surg. 2000, 70:816-817.

14. Michon J: Phlegmon of the tendon sheaths . Ann Chir. 1974, 28:277-280.

15. Young-afat DA, Dayicioglu D, Oeltjen JC, Garrison AP: Fishing-injury-related flexor tenosynovitis of the hand: a case report and review. Case Rep Orthop. 2013, 2013:587176.

16. Khashe S, Janda JM: Biochemical and pathogenic properties of Shewanella alga and Shewanella putrefaciens. J Clin Microbiol. 1998, 36:783-787.

17. Pang HN, Teoh LC, Yam AK, Lee JY, Puhaindran ME, Tan AB: Factors affecting the prognosis of pyogenic flexor tenosynovitis. J Bone Joint Surg Am. 2007, 89:1742-1748. 10.2106/JBJS.F.01356 